\title{
Adaptación del Cuestionario de Capital Psicológico al ámbito educativo en una muestra argentina
}

\section{Adaptation of the Psychological Capital Questionnaire in the Educational Context in an Argentine Sample}

\section{Fátima Soledad Schönfeld*}

Centro Interdisciplinario de Investigaciones en Psicología Matemática y Experimental "Dr. Horacio

J. A. Rimoldi", Consejo Nacional de Investigaciones Científicas y Técnicas, Buenos Aires,

Argentina

Pontificia Universidad Católica Argentina, Facultad de Psicología y Psicopedagogía, Buenos Aires, Argentina

ORCID: https://orcid.org/0000-0002-5395-3118

\section{Belén Mesurado}

Centro Interdisciplinario de Investigaciones en Psicología Matemática y Experimental "Dr. Horacio

J. A. Rimoldi", Consejo Nacional de Investigaciones Científicas y Técnicas, Buenos Aires,

Argentina

Universidad Austral, Buenos Aires, Argentina

ORCID: https://orcid.org/0000-0002-5907-5854

Recibido 29-04-19 Revisado 30-05-19 Aprobado 04-11-19 En línea 11-11-19

*Correspondencia

Email: fatischonfeld@hotmail.com
Citar como:

Schönfeld, F., \& Mesurado, B. (2020). Adaptación del Cuestionario de Capital Psicológico al ámbito educativo en una muestra argentina. Propósitos y Representaciones, 8(1), e315. doi: http://dx.doi.org/10.20511/pyr2020.v8n1.315

() Universidad San Ignacio de Loyola, Vicerrectorado de Investigación, 2020. 


\section{Resumen}

El capital psicológico es definido como un estado de desarrollo psicológico positivo que representa la valoración positiva que la persona hace de las circunstancias y su probabilidad de éxito basada en el esfuerzo y la perseverancia. Se trata de un constructo compuesto por cuatro componentes o fortalezas psicológicas: eficacia, optimismo, esperanza y resiliencia. El mismo fue ampliamente descripto y evaluado en el ámbito laboral; sin embargo, puede aplicarse a otras esferas de la vida de una persona, como por ejemplo al ámbito educacional. De allí que el objetivo del presente trabajo es evaluar las propiedades psicométricas de la adaptación al contexto educativo del Cuestionario de Capital Psicológico en población adolescente Argentina $(\mathrm{N}=313)$. Se analizará la validez de constructo, la validez convergente (con rendimiento escolar y engagement) y los niveles de fiabilidad de dicha adaptación. Los resultados hallados aportan evidencias empíricas que avalan buenos niveles de validez y confiabilidad del Cuestionario de Capital Psicológico en el ámbito educativo y en la población adolescente.

Palabras clave: Capital psicológico; Psicometría; Educación; Adolescentes.

\section{Summary}

Psychological capital is defined as a state of positive psychological development that represents the person's positive assessment of the circumstances and their probability of success based on effort and perseverance. It is a construct composed by four components or psychological strengths: efficacy, optimism, hope and resilience. It was widely described and evaluated in the workplace; however, psychological capital can be applied to other spheres of a person's life, such as education. Hence, the objective of this study is to evaluate the psychometric properties of the adaptation to the educational context of the Psychological Capital Questionnaire in the Argentinean adolescent population $(\mathrm{N}=$ 313). Construct validity, convergent validity (with school performance and engagement) and reliability levels are analyzed. This research provides empirical evidence that supports good levels of validity and reliability of the Psychological Capital Questionnaire in the educational context in adolescent population.

Keywords: Psychological Capital; Psychometric; Education; Adolescents.

\section{Introducción}

La Psicología Positiva es una línea teórica que puso su foco de interés en aquellos aspectos funcionales del desarrollo de las personas, centrándose en las fortalezas y en el funcionamiento adaptativo más que en los aspectos disfuncionales (Seligman \& Csikszentmihalyi, 2000). Además, dicha disciplina estudia el modo en que el ser humano puede crecer y prosperar a lo largo de su vida (Snyder \& López, 2002, Mesurado, 2017). Uno de los constructos desarrollados en el marco de la Psicología Positiva es el de Capital Psicológico.

El capital psicológico es definido como un estado de desarrollo psicológico positivo, que representa la valoración positiva que la persona hace de las circunstancias y su probabilidad de éxito basada en el esfuerzo y la perseverancia (Azanza, Domínguez, Moriano \& Molero, 2014; Luthans, Avolio, Avey \& Norman, 2007). 
Se trata de un constructo compuesto por cuatro componentes que refieren a cuasi-estados o fortalezas psicológicas: la eficacia, el optimismo, la esperanza y la capacidad de resiliencia. Desde el enfoque cognitivo social de Bandura (1986) se define a la autoeficacia como la confianza que la persona experimenta en relación con sus capacidades para fomentar la motivación, los recursos cognitivos y la acción necesaria para ejecutar con éxito una tarea específica en un contexto determinado (Stajkovic \& Luthans, 1998). En relación al optimismo, Seligman y colaboradores encontraron que las expectativas hacia el futuro están relacionadas con la explicación que la persona hace acerca de los eventos. Los estilos explicativos pueden ser atribuidos a causas internas o externas, explicaciones estables o inestables, globales o específicas (Seligman, Reivich, Jaycox \& Gillham, 1999). El optimismo es la expectativa de que las cosas van a salir bien y está vinculado con un estilo de atribución que explica los sucesos positivos como personales, permanentes y globales y los eventos negativos como externos, temporales y específicos (Seligman, 1998); por tanto, el optimismo implica hacer atribuciones positivas sobre el éxito presente y futuro (Azanza, et al., 2014; Luthans, et al, 2007). La esperanza es un estado positivo de motivación que se basa, por un lado, en contar con la energía para alcanzar un objetivo concreto, y por el otro, en la capacidad de planificar las vías para llegar al mismo; implica ser perseverante en el cumplimiento de metas y, de ser necesario, reorientar las trayectorias de estas (Azanza et al., 2014; Luthans, et al., 2007). De esta manera, la esperanza no sólo incluye la determinación y motivación para alcanzar algo, sino también un plan viable para llevar a cabo exitosamente la tarea en pos de alcanzar el objetivo (Luthans, Luthans \& Norman, 2005). Por último, la resiliencia se refiere a la capacidad de recuperarse frente a situaciones adversas y salir fortalecido de ellas (Azanza, et al., 2014; Luthans, et al, 2007). Avey, Patera y West (2006) la definen como un sistema adaptativo que permite al individuo recuperarse rápidamente de un inconveniente o un fracaso. La investigación ha demostrado que las personas pueden aprender a ser resilientes (Youssef, 2004).

El capital psicológico es un fenómeno que va más allá del capital humano y social, aludiendo al "quién soy" de una persona (Luthans, Luthans \& Luthans, 2004). Diversos estudios realizados en el ámbito laboral demostraron que aquellos empleados con un alto nivel de capital psicológico (considerado juntamente con sus cuatro dimensiones), presentaban una mayor satisfacción y un mejor rendimiento que aquellos que exhibían altos niveles de autoeficacia, optimismo, esperanza o resiliencia por separado (Luthans, et al., 2007). Por otra parte, Luthans, Norman, Avolio \& Avey (2008) encontraron una relación positiva entre el capital psicológico y el rendimiento en una muestra de estudiantes y trabajadores. Otra investigación evidenció que una persona con altos niveles de capital psicológico logra experimentar compromiso y que a su vez dicho compromiso contribuye a incrementar aún más su nivel de capital psicológico (Sweetman \& Luthans, 2010). En este sentido, Luthans, Youssef, Sweetman, y Harms (2013) han propuesto un circuito de positividad en el que el capital psicológico, como un estado de desarrollo psicológico positivo, lleva a experimentar mejores y mayores niveles de salud y bienestar general, lo cual, a su vez, refuerza los recursos psicológicos positivos (como, por ejemplo, el capital psicológico).

Otras investigaciones publicadas en los últimos años coinciden respecto a la asociación positiva entre el capital psicológico, la salud y el bienestar (Siu, 2015; Williams, Kern y Waters, 2015). Por otra parte, ciertos estudios hacen referencia a una relación negativa entre el capital psicológico y los problemas de salud mental, el abuso de sustancias, como así también los conflictos en la vida laboral y privada, teniendo el capital psicológico una función preventiva de dichos problemas (Krasikova, Lester \& Harms, 2015; Karatepe \& Karadas, 2015; Martinez-Corts, Demeroutti, Bakker \& Boz, 2015; Siu, 2015). 
Se ubica al capital psicológico entre un estado puro y un rasgo, debido a que no es tan cambiante como el primero ni tan fijo como el segundo (Luthans, Avey, Avolio \& Peterson, 2010). En este sentido, sería un estado abierto al desarrollo, al que se puede llegar y mantener de manera relativamente estable (Luthans, et al., 2007). En relación con dicha posibilidad de desarrollo, hay evidencia preliminar de que el capital psicológico puede ser promovido a través de intervenciones cortas y focalizadas llevadas a cabo en diversos ámbitos (Luthans, Avey, Avolio, Norman \& Combs, 2006; Luthans, Avey \& Patera, 2008; Luthans, et al., 2007; Luthans, et al., 2010).

El constructo capital psicológico fue ampliamente descripto y evaluado en el ámbito laboral. Sin embargo, por ser un estado psicológico caracterizado por la valoración optimista de las circunstancias y de la probabilidad de éxito fundada en el esfuerzo y la perseverancia, es que puede aplicarse a otras esferas de la vida de una persona, como por ejemplo al ámbito educacional. En el contexto educativo, los estudiantes pueden enfrentar diferentes situaciones adversas, como, por ejemplo, la dificultad de comprender y aprender ciertos contenidos, la presión de cumplir con plazos y entregas, conflictos en las relaciones interpersonales con los compañeros de estudio o con los docentes, etc. Ante estas circunstancias, resultaría beneficioso que los estudiantes tuvieran altos niveles de capital psicológico. De hecho, actitudes optimistas y esperanzadas frente a estas situaciones problemáticas pueden ser una buena vía de acción para alcanzar el éxito académico. Asimismo, la percepción de eficacia es un recurso que suele contribuir a la perseverancia en la educación y es un factor predictor del compromiso académico (Mesurado, Richaud \& Mateo, 2016). Por otro lado, estas situaciones adversas pueden generar en los adolescentes actitudes resilientes frente a la tarea educativa o las actividades vinculadas a ella, de tal modo que se constituyen en oportunidades para salir fortalecidos. De allí que sería esencial contar con un instrumento que permita evaluar el capital psicológico de los estudiantes, específicamente en el ámbito académico.

El objetivo del presente trabajo es evaluar las propiedades psicométricas de la adaptación al contexto educativo del Cuestionario de Capital Psicológico (Psycap) (Psychological Capital Questionnaire de Luthans, Avolio, Avey \& Norman, 2007) en población adolescente Argentina. Específicamente se analizará la validez de constructo, la validez convergente (con rendimiento escolar y engagement) y los niveles de fiabilidad de la adaptación del Psycap al contexto educativo.

\section{Método}

\section{Participantes y procedimiento.}

Los participantes de esta investigación fueron seleccionados a través de un muestreo no probabilístico, de tipo intencional. Dicha muestra se conformó por 313 adolescentes de los cuales el $68 \%$ eran mujeres $(n=213)$ y el $32 \%$ varones $(n=100)$. Los sujetos eran estudiantes de nivel secundario que, al momento de la evaluación, cursaban $1^{\circ}, 2^{\circ}$ o $3^{\circ}$ año del Ciclo Básico Común en escuelas públicas de gestión privada de la provincia de Entre Ríos, Argentina. Las edades de los adolescentes se encontraban comprendidas entre los 12 y los 16 años, observándose una media de edad de 13.46 $(\mathrm{DT}=1.05)$.

Conformaron la muestra estudiante de dos escuelas entrerrianas: "Instituto Santa María" D8 de la ciudad de Diamante e Instituto "Sagrado Corazón" D-5 de la ciudad de Crespo. Dichas instituciones convocan a estudiantes de diferentes sectores socio-económicos, prevaleciendo el nivel medio. En relación a la cantidad de alumnos por año, constituyeron la muestra del presente trabajo

Propósitos y Representaciones

Ene. - Abr. 2020, Vol. 8, N 1 , e315

http://dx.doi.org/10.20511/pyr2020.v8n1.315 
un total de 105 estudiantes de $1^{\circ}$ año, 101 adolescentes de $2^{\circ}$ año y 107 de $3^{\circ}$ año del Ciclo Básico Común.

Para realizar las evaluaciones se estableció previamente el contacto con las autoridades de las instituciones educativas y se solicitó de manera escrita el consentimiento informado por parte de los padres o tutores de los alumnos. Las administraciones fueron efectuadas en forma grupal, durante el horario de clases. Se tomaron en cuenta las correspondientes consideraciones éticas para garantizar el resguardo de la confidencialidad de los datos obtenidos.

\section{Instrumentos.}

\section{Cuestionario de Capital Psicológico para el contexto educacional.}

Se modificaron los ítems de la versión abreviada del Psychological Capital Questionnaire desarrollado por Luthans, Avolio, Avey y Norman (2007), contextualizándolos al ámbito educacional. El instrumento original consta de 12 ítems que evalúan las cuatro dimensiones del capital psicológico: autoeficacia (3 ítems; ej. ítem original: "Me siento seguro representando mi área de trabajo en las reuniones con la dirección o gerencia", ej. ítem modificado: "Me siento seguro si tengo que representar a mi curso en alguna actividad de la escuela"), esperanza (4 ítems; ej. ítem original: "Si me encuentro con un problema en el trabajo, puedo pensar muchas maneras de salir de él", ej. ítem modificado: "Si me encuentro con un problema en la escuela, puedo buscar diferentes maneras de resolverlo"), resiliencia (3 ítems; ej. ítem original: "Puedo atravesar las situaciones difíciles en el trabajo porque he experimentado dificultades antes", ej. ítem modificado: "Puedo enfrentar situaciones complicadas en la escuela porque me han pasado cosas similares antes y pude resolverlas") y optimismo ( 2 ítems; ej. ítem original: "Siempre miro el lado bueno de las cosas relacionadas con mi trabajo", ej. ítem modificado: "Siempre miro el lado bueno de las cosas de la escuela"). Los estudiantes debían evaluar las afirmaciones utilizando una escala tipo Likert que va de 1 ("Muy en desacuerdo") a 6 ("Muy de acuerdo") puntos.

\section{Utrecht-work Compromiso Scale for Students (UWES-S).}

Para medir el compromiso de los estudiantes con su tarea educativa se utilizó la adaptación argentina realizada por Mesurado, Richaud y Mateo (2016) del Utrecht-Work Compromiso Scale (UWES) en su versión para estudiantes (Schaufeli, Martínez, Marques Pinto, Salanova y Bakker, 2002). Es un auto-reporte que consta de 14 ítems, que miden las tres dimensiones del compromiso: vigor (5 ítems, ej.: "Cuando me levanto por la mañana tengo ganas de ir a clase"), dedicación (5 ítems, ej.: "Estoy orgulloso de mis estudios") y absorción (4 ítems, ej.: "El tiempo vuela cuando estoy estudiando"). A través de una escala Likert que va de 0 ("Nunca") a 6 ("Siempre") puntos, los estudiantes debían responder cómo se sienten respecto a sus estudios.

\section{Rendimiento académico.}

Como indicador del rendimiento académico de los estudiantes evaluados, se relevaron los promedios generales anuales de cada uno de ellos. Los mismos fueron solicitados a las instituciones escolares, las cuales brindaron acceso a dicha información, registrada en los libros de actas matrices. 


\section{Procedimiento estadístico.}

Para evaluar la validez de constructo del Cuestionario de Capital Psicológico para el contexto educacional se realizó un Análisis Factorial Confirmatorio evaluando la presencia de las dimensiones propuestas por los autores originales. Se utilizó el método de máxima verosimilitud para estudiar el ajuste del modelo, teniendo en cuenta el valor chi cuadrado, chi/gl, los índices de bondad de ajuste CFI (Comparative Fit Index), GFI (Goodness of Fit Index) y AGFI (Adjusted Goodness of Fit Index); y los índices de error RMR (Root Mean Residual) y RMSEA (Root Mean Squared Error of Aproximation). Se empleó para ello el programa AMOS, en su versión 21. Para estudiar la validez convergente de la adaptación del cuestionario, se utilizó análisis de correlación $r$ de Pearson. Para evaluar la consistencia interna del instrumento se utilizó el coeficiente Omega (McDonald's Omega), calculando los índices para la escala global como así también para cada una de las dimensiones.

\section{Resultados}

\section{Análisis descriptivo}

Se realizaron análisis de estadística descriptiva básica calculando los valores mínimos y máximos, medias y desvíos típicos para la presente muestra. Por otra parte, se calcularon los índices de asimetría y curtosis, los cuales se encuentran dentro de los límites esperados (ver Tabla 1). A su vez, se analizaron las propiedades de normalidad de la adaptación de la escala, utilizando el índice de Normalidad Multivariada, el cual obtuvo un puntaje de 20,22.

\section{Tabla 1.}

Estadísticos descriptivos Cuestionario de Capital Psicológico (N=313)

\begin{tabular}{ccccccc}
\hline & Mín & Máx & M & DE & Asimetría & Curtosis \\
\hline Ítem1 & 1,00 & 6,00 & 4,25 & 1,41 &,- 63 &,- 38 \\
Ítem 2 & 1,00 & 6,00 & 4,15 & 1,30 &,- 53 &,- 22 \\
Ítem 3 & 1,00 & 6,00 & 4,28 & 1,53 &,- 70 &,- 52 \\
Ítem 4 & 1,00 & 6,00 & 4,27 & 1,34 &,- 53 &,- 28 \\
Ítem 5 & 1,00 & 6,00 & 3,90 & 1,41 &,- 40 &,- 68 \\
Ítem 6 & 1,00 & 6,00 & 4,26 & 1,25 &,- 57 &,- 01 \\
Ítem 7 & 1,00 & 6,00 & 4,02 & 1,43 &,- 44 &,- 59 \\
Ítem 8 & 1,00 & 6,00 & 4,23 & 1,39 &,- 60 &,- 33 \\
Ítem 9 & 1,00 & 6,00 & 3,62 & 1,52 &,- 11 & $-1,09$ \\
Ítem 10 & 1,00 & 6,00 & 4,20 & 1,46 &,- 60 &,- 45 \\
Ítem 11 & 1,00 & 6,00 & 3,84 & 1,46 &,- 22 &,- 73 \\
Ítem 12 & 1,00 & 6,00 & 4,30 & 1,47 &,- 75 &,- 20 \\
\hline
\end{tabular}

\section{Validez de estructura.}

Se comprobó la estructura factorial del Cuestionario de Capital Psicológico aplicado en estudiantes de nivel secundario, por medio de un Análisis Factorial Confirmatorio, utilizando el método de la máxima verosimilitud, dado que se encontró una normalidad multivariada en los datos. Por medio de dicho procedimiento se obtuvo un índice chi cuadrado de 96,70 siendo este valor estadísticamente 
significativo ( $p \leq .001)$. El puntaje obtenido en chi/gl fue de 2,01. Por otra parte, el GFI arrojó un valor de .95 , el AGFI una puntuación de .92 y CFI de .93. Dado que todos los valores están por encima de .90 se concluye que se encontró un buen ajuste del modelo a los datos obtenidos. Respecto a la medición del error, el índice RMR arrojó un puntaje de .11 y el RMSEA obtuvo un valor de .05, indicando que los índices de error están mínimamente elevados. El pesaje de los ítems en cada factor puede observarse en la figura 1.

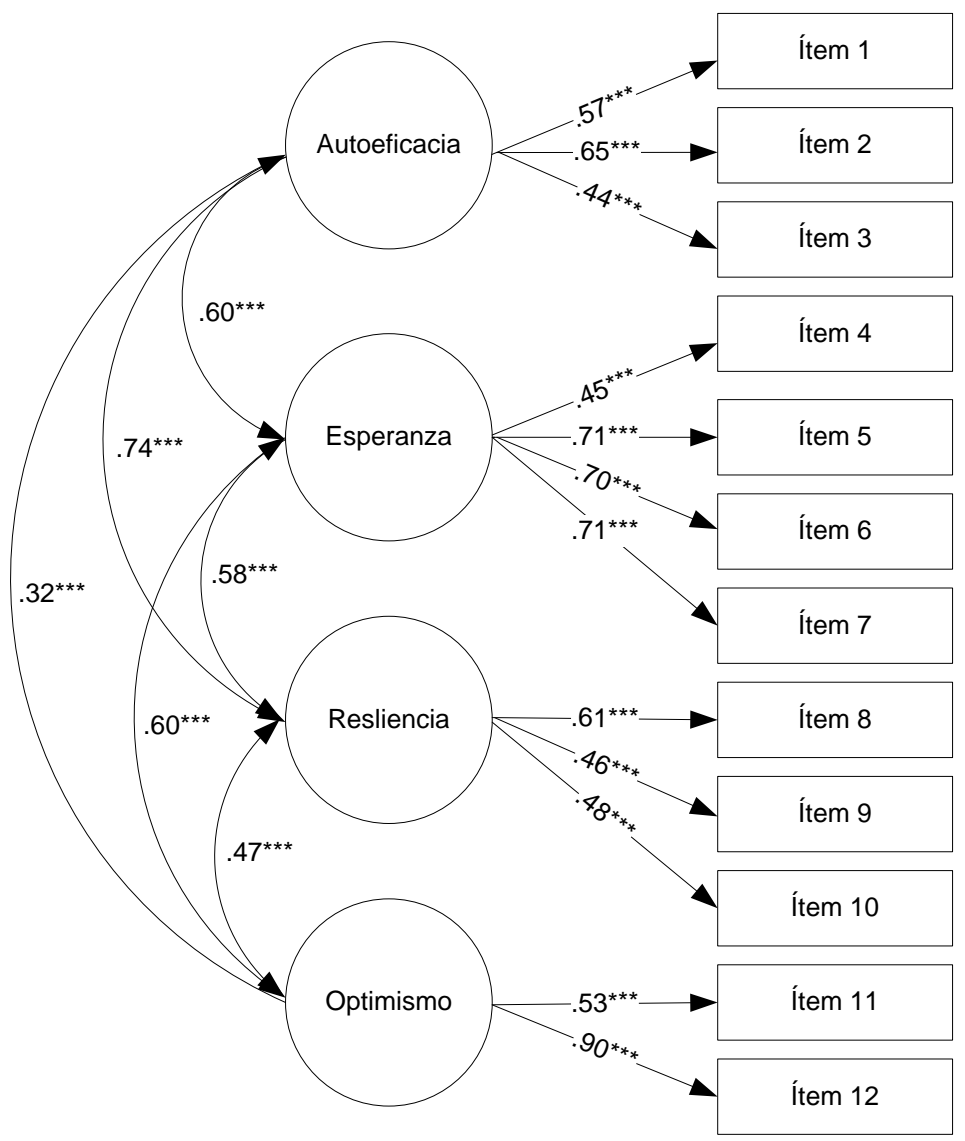

Nota: $* * * \mathrm{p}<.001 ; * * \mathrm{p}<.01 ; * \mathrm{p}<.05$

Figura 1. Cargas factoriales estandarizadas y correlaciones entre dimensiones del Cuestionario de capital Psicológico en el contexto educativo

\section{Validez convergente.}

Con el objetivo de analizar la validez convergente del Cuestionario de Capital Psicológico para el contexto educacional se estudió su relación con el compromiso académico y con el promedio del alumno como indicador de rendimiento académico. Los resultados indican que hay una correlación positiva entre las 4 dimensiones del capital psicológico (autoeficacia, esperanza, resiliencia y optimismo) y las tres dimensiones del engagement (vigor, dedicación y absorción), así como con el rendimiento académico. 
Tabla 2.

Coeficientes de correlación de Pearson. Asociación entre los componentes de Capital Psicológico, las dimensiones de Engagement y el rendimiento académico $(N=313)$.

\begin{tabular}{ccccc}
\hline & Autoeficacia & Esperanza & Resiliencia & Optimismo \\
\hline Vigor &, $365^{* *}$ &, $569^{* *}$ &, $291^{* *}$ &, $888^{* *}$ \\
Dedicación &, $279^{* *}$ &, $528^{* *}$ &, $260^{* *}$ &, $862^{* *}$ \\
Absorción &, $294^{* *}$ &, $496^{* *}$ &, $249^{* *}$ &, $856^{* *}$ \\
Promedio &, $259^{* *}$ &, $436^{* *}$ &, $175^{* *}$ &, $133^{*}$ \\
\hline
\end{tabular}

\section{Consistencia interna de la prueba.}

Para examinar la consistencia interna de la adaptación del cuestionario se utilizó el coeficiente Omega McDonald, dado que resulta más robusto que el índice Alfa de Cronbach (McNeish, 2017). De este modo, la escala considerada de manera global obtuvo un Omega de .93. La dimensión de autoeficacia arrojó un Omega de .74, la de esperanza un índice de .81, la de resiliencia de .73 y la de optimismo de .81 .

\section{Discusión}

El análisis de validez de la adaptación del Cuestionario de Capital Psicológico al ámbito educativo en lengua española ha mostrado una estructura factorial idéntica a la escala original de capital psicológico en el ámbito laboral (Luthans, Avolio, Avey \& Norman, 2007). Así mismo, mediante el Análisis Factorial Confirmatorio se han podido identificar los cuatro componentes propuestos por los autores originales de la escala: autoeficacia, optimismo, esperanza y resiliencia.

Respecto a la validez convergente del Cuestionario de Capital Psicológico para el contexto educacional, los resultados indican, tal como esperábamos, que esta variable se encuentra relacionada con el compromiso académico de los estudiantes evaluados. En relación a ello, un estudio llevado a cabo por Sweetman y Luthans (2010) evidenció que una persona que experimente altos niveles de capital psicológico logra alcanzar altos niveles de compromiso y que, a su vez, dicho compromiso contribuye a incrementar su nivel de capital psicológico. De esta manera, un individuo que cree en sus propias capacidades, que es optimista en cuanto a la posibilidad de poder alcanzar resultados exitosos, que desarrolla esperanza frente a la adversidad y se vuelve resiliente; es capaz de experimentar el compromiso en cualquier tarea que realice. El argumento de que el capital psicológico es un factor predictivo del engagement académico podría ser explicado por la idea de que determinados recursos personales contribuyen a transformar las demandas académicas en desafíos (Bakker \& Demerouti, 2008). De esta manera, dichos recursos ayudan al estudiante a sentirse motivado, concentrarse, dedicarse y estar absorbido en su trabajo (Bakker, Schaufeli, Leiter \& Taris, 2008). Además de los antecedentes nombrados anteriormente, otro estudio llevado a cabo por Siu, Bakker y Jiang (2014), encontró una asociación entre el capital psicológico, el compromiso académico y la motivación intrínseca en estudiantes universitarios.

Por otra parte, los datos obtenidos permiten afirmar que el capital psicológico se encuentra relacionado con el rendimiento de los estudiantes. Esto coincide con lo propuesto por Luthans y sus colaboradores (2008), quienes encontraron una asociación de carácter positivo entre el capital 
psicológico y el rendimiento en una muestra de estudiantes y trabajadores. En relación a esto último, diversas investigaciones precedentes evidencian el impacto positivo del capital psicológico sobre el rendimiento académico de los estudiantes (Jafri, 2013; Luthans, Luthans \& Jensen, 2012; Malone, 2010; Tjakraatmadja \& Febriansyah, 2007; Vanno, Kaemkate \& Wongwanich, 2014). Si bien el número de estudios que establecen esta vinculación entre variables es limitado, los hallazgos existentes indican que el capital psicológico tiene una influencia significativa positiva en el rendimiento académico de los estudiantes.

Otro aspecto de relevancia en el proceso de adaptación de una escala es el estudio de la confiabilidad de esta. Dado que la prueba de consistencia interna utilizada habitualmente (Alfa de Cronbanch) está afectada por el número de ítems que componen la dimensión, en este estudio se utilizó el coeficiente Omega. El coeficiente Omega McDonalds presenta tres ventajas sobre el Alfa de Cronbanch: 1. Se calcula a partir de las cargas factoriales, 2. No depende del número de ítems y 3. Es resiste al principio de equivalencia (Ventura-León \& Caycho-Rodríguez, 2017). Los análisis de confiabilidad realizados en el presente trabajo indicaron que la adaptación de la escala al contexto educativo ha mostrado tener buenos índices tanto para la escala global, como para cada una de las dimensiones que la componen.

En definitiva, las propiedades psicométricas de la adaptación realizada son adecuadas. De esta manera, los resultados hallados aportan evidencias empíricas que avalan buenos niveles de validez y confiabilidad del Cuestionario de Capital Psicológico en el ámbito educativo y en la población adolescente.

Teniendo en cuenta que el capital psicológico se encuentra relacionado al a la salud y al bienestar de los seres humanos y que, por otra parte, tiene posibilidades de ser desarrollado, se vuelve importante destacar la necesidad de fomentar dicho estado positivo en las personas.

Los constructos de auto-eficiencia, optimismo, esperanza y resiliencia definen estados positivos que se vinculan con la capacidad de responder con confianza y optimismo ante las eventualidades que se le pueden presentar a una persona. Refieren a las evaluaciones que un sujeto realiza sobre los recursos cognitivos y emocionales que posee en un momento determinado y de cómo los puede aplicar ante cualquier circunstancia (Luthans, et al., 2010). Así, en el ámbito escolar, los adolescentes se ven cotidianamente involucrados en circunstancias que se constituyen para ellos en demandas y desafíos, ante las cuales deben responder con sus recursos personales.

De este modo, el capital psicológico engloba habilidades que pueden favorecer el desarrollo psicológico de los adolescentes en el ámbito escolar. Dicho desarrollo puede verse plasmado en un mayor compromiso con la actividad escolar, como así también en un mejor rendimiento académico. Así, adquiere relevancia el hecho de disponer de un instrumento de evaluación del capital psicológico adaptado al contexto educacional, como herramienta diagnóstica que permita conocer la presencia y los niveles de estas capacidades psicológicas en los estudiantes de nivel secundario.

Por último, el hecho de contar con un cuestionario adaptado a la mencionada población, facilita no sólo el diagnóstico de los niveles de capital psicológico sino también, teniendo en cuenta dicho diagnóstico, la posibilidad de realizar intervenciones para favorecer el desarrollo de estas cualidades. Como se dijo con anterioridad, existe evidencia que confirma que la auto-eficacia, el optimismo, la esperanza y la resiliencia pueden ser facilitadas a través de intervenciones cortas y focalizadas (Gutiérrez, 2016). De este modo, podría pensarse en la posibilidad de implementar algún tipo de intervención en el contexto del aula, con el objetivo de promover el capital psicológico. 


\section{Limitaciones y futuras investigaciones.}

En cuanto a las limitaciones del presente estudio, es necesario destacar que la selección de los sujetos participantes fue no aleatoria, de modo que los resultados podrían estar afectados por las características de la muestra de estudiantes incluidos en el estudio. En el futuro, sería importante estudiar la variable extendiendo el número de sujetos participantes. Por otro lado, podría ser interesante ampliar los estudios de validez convergente del Cuestionario de Capital Psicológico para el contexto educativo, analizando su relación con otras variables tales como la salud y el bienestar de los estudiantes en el contexto áulico. Además, sería de gran interés analizar otro aspecto de la confiabilidad de la escala como por ejemplo la estabilidad de sus puntuaciones, realizando un análisis de test-retest.

\section{Referencias}

Avey, J., Patera, J., \& West, B. (2006). The implications of positive psychological capital on employee absenteeism. Journal of Leadership and Organizational Studies, 13, 42 - 60. doi: https://doi.org/10.1177/10717919070130020401

Azanza, G., Domínguez, A., Moriano, J., \& Molero, F. (2014). Capital psicológico positivo. Validación del cuestionario PCQ en España. Anales de Psicología, 30(1), 294-301.

Bakker, A. B., \& Demerouti, E. (2008). Towards a model of work engagement. Career Development International, 13, 209-223. Doi: https://doi.org/10.1108/13620430810870476

Bakker, A. B., Schaufeli, W. B., Leiter, M. P. y Taris, T. W. (2008). Work engagement: an emerging concept in occupational health psychology. Work and Stress, 22, 187-200.

Bandura, A. (1986). Social foundations of thought and action: A social cognitive theory. Englewood Cliffs, NJ: Prentice- Hall.

Jafri, M. H. (2013). A study of the relationship of psychological capital and students' performance. Business Perspectives and Research, 1(2), 9-16. doi: https://doi.org/10.1177/2278533720130202

Karatepe, O. M., \& Karadas, G. (2015). Do psychological capital and work engagement foster frontline employees' satisfaction? A study in the hotel industry. International Journal of Contemporary Hospitality Management, 27, 1254-1278. doi: http://dx.doi.org/10.1108/IJCHM-01-2014-0028

Krasikova, D. V., Lester, P. B., \& Harms, P. D. (2015). Effects of psychological capital on mental health and substance abuse. Journal of Leadership and Organizational Studies, 22, 280-291. doi: http://dx.doi.org/10.1177/1548051815585853

Luthans, B. C., Luthans, K. W., \& Jensen, S. M. (2012). The impact of business school students' psychological on academic performance. Journal of Education for Business, 87, 253-259.

Luthans, B., Luthans, K., \& Norman, S. (2005). The proposed contagion effect of hopeful leaders on the resiliency of employees and organizations. Journal of Leadership and Organizational Studies, 12, 55 - 64. doi: https://doi.org/10.1177/107179190501200205

Luthans, F., Avey, J., \& Patera, J. (2008). Experimental analysis of a web-based training intervention to develop positive psychological capital. Academy of Management Learning and Education, 7(2), 209-221. doi: https://doi.org/10.5465/amle.2008.32712618

Luthans, F., Avey, J., Avolio, B. y Peterson, S. (2010). The development and resulting performance impact of positive psychological capital. Human Resource Development Quarterly, 21(1), 4167. doi: https://doi.org/10.1002/hrdq.20034

Luthans, F., Avey, J., Avolio, B., Norman, S., \& Combs, G. (2006). Psychological capital development: toward a micro-intervention. Journal of Organizational Behavior, 27, 387-393. doi: https://doi.org/10.1002/job.373

Propósitos y Representaciones

Ene. - Abr. 2020, Vol. 8, $\mathrm{N}^{\circ} 1$, e315

http://dx.doi.org/10.20511/pyr2020.v8n1.315 
Luthans, F., Avolio, B., Avey, J., \& Norman, S. (2007). Positive psychological capital: measurement and relationship with performance and satisfaction. Personnel Psychology, 60, 541-572.

Luthans, F., Luthans, K. W., \& Luthans, B. C (2004). Positive psychological capital: beyond human and social capital. Business Horizons, 47(1), 45-50.

Luthans, F., Norman, S. M., Avolio, B. J., \& Avey, J. B. (2008). The mediating role of psychological capital in the supportive organizational climate-employee performance relationship. Journal of Organizational Behavior, 29(2), 219-238. doi : https://doi.org/10.1002/job.507

Luthans, F., Youssef, C. M., Sweetman, D. S., \& Harms, P. D. (2013). Meeting the leadership challenge of employee well-being through relationship PsyCap and health PsyCap. Journal of Leadership and Organizational Studies, 20, 118-133. doi: https://doi.org/10.1177/1548051812465893

Malone, L. D. (2010). Individual differences and stress reactions as predictors of performance in pilot trainees (Doctoral dissertation, Kansas State University).

Martinez-Corts, I., Demerouti, E., Bakker, A. B., \& Boz, M. (2015). Spillover of interpersonal conflicts from work into nonwork: A daily diary study. Journal of Occupational Health Psychology, 20, 326-337. doi: http://dx.doi.org/10.1037/a0038661

McNeish, D. (2017). Thanks coefficient alpha, we'll take it from here. Psychological Methods. http://dx.doi.org/10.1037/met0000144

Mesurado, B. (2017). Psicología positiva. En A. Vanney, I. Silva, y J. F. Franck (Eds.), Diccionario Interdisciplinar Austral. Buenos Aires: Universidad Austral. Disponible en http://dia.austral.edu.ar/Psicología_positiva

Mesurado, B., Richaud, M.C, \& Mateo, J. N. (2016). Engagement, flow, self-efficacy, and eustress of university students: a cross-national comparison between the Philippines and Argentina. The Journal of Psychology, 150(3), 281-299.

Gutiérrez, J. C. P. (2016). Evaluación De Una Práctica Para El Desarrollo Del Capital Psicológico. Orbis. Revista Científica Ciencias Humanas, 12(34), 4-25.

Schaufeli, W. B., Martínez, I., Marqués-Pinto, A., Salanova, M., \& Bakker, A. (2002). Burnout and engagement in university students: a cross-national study. Journal of Cross-cultural Studies, 33, 464-481.

Seligman, M. E. P. (1998). Learned optimism. New York: Pocket Books.

Seligman, M. E., \& Csikszentmihalyi, M. (2000). Positive psychology. An Introduction. American Psychologist, 55, 5-14.

Seligman, M., Reivich, K, Jaycox, L. y Gillham, J. (1999). Niños optimistas. Barcelona: Grijalbo.

Siu, O. L. (2015). Psychological capital, work well-being, and work-life balance among Chinese employees. Journal of Personnel Psychology, 12, 170-181. doi: http://dx.doi.org/10.1027/1866-5888/a000092

Siu, O. L., Bakker, A. B., \& Jiang, X. (2014). Psychological capital among university students: relationships with study engagement and intrinsic motivation. Journal of Happiness Studies, 15(4), 979-994.

Snyder, C., \& Lopez Shane (Eds.). Handbook of positive psychology. Oxford, U.K.: Oxford UniversityPress.

Stajkovic, A. D., \& Luthans, F. (1998). Social cognitive theory and self-efficacy: going beyond traditional motivational and behavioral approaches. Organizational Dynamics, 26, 62 - 74. doi: https://doi.org/10.1016/S0090-2616(98)90006-7

Sweetman, D., \& Luthans, F. (2010). The power of positive psychology: psychological capital and work compromiso. In A. B. Bakker y M. P. Leiter (Eds.), Work compromiso: A handbook of essential theory and research (pp. 54-68). Hove, East Sussex: Psychology Press. 
Tjakraatmadja, J. H., \& Febriansyah, H. (2007). The influence of psychological capital and learning environment toward SBM-ITB students' GPA. Indonesian Journal for the Science of Management, 6, 1-13.

Vanno, V., Kaemkate, W., \& Wongwanich, S. (2014). Relationships between academic performance, perceived group psychological capital, and positive psychological capital of Thai undergraduate students. Procedia-Social and Behavioral Sciences, 116, 3226-3230.

Ventura-León, J. L., \& Caycho-Rodríguez, T. (2017). El coeficiente omega: un método alternativo para la estimación de la confiabilidad. Revista Latinoamericana de Ciencias Sociales, Niñez y Juventud, 15(1), 625-627.

Youssef, C. (2004). Resilience development of organizations, leaders and employees: multi-level theory building and individual- level, path-analytical empirical testing. Ph.D Dissertation: University of Nebraska. 